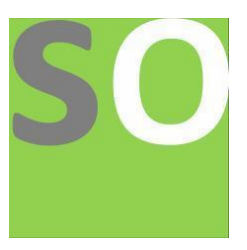

Article title: Chronology protection conjecture may not hold

Authors: Wen-Xiang Chen[1]

Affiliations: South China Normal University[1]

Orcid ids: 0000-0002-0560-8280[1]

Contact e-mail: 954763891@qq.com

License information: This work has been published open access under Creative Commons Attribution License http://creativecommons.org/licenses/by/4.0/, which permits unrestricted use, distribution, and reproduction in any medium, provided the original work is properly cited. Conditions, terms of use and publishing policy can be found at https://www.scienceopen.com/.

Preprint statement: This article is a preprint and has not been peer-reviewed, under consideration and submitted to ScienceOpen Preprints for open peer review.

DOI: 10.14293/S2199-1006.1.SOR-.PPOX9US.v1

Preprint first posted online: 09 July 2020

Keywords: Chronology protection conjecture, entropy reduction, naked singularity 


\title{
Chronology protection conjecture may not hold
}

\author{
Wen-Xiang Chen* \\ Institute of quantum matter, \\ School of Physics and Telecommunication Engineering, \\ South China Normal University, \\ Guangzhou 510006, China
}

\begin{abstract}
The cosmic censorship hypothesis has not been directly verified, and some physicists also question the validity of the cosmological censorship hypothesis. Through theoretical prediction, it pointed out that the existence of naked singularities is possible. This article puts forward a point that, under the quantum gravity equation, although there is no concept of wormholes, the chronology protection conjecture may not be true.
\end{abstract}

Keywords: Chronology protection conjecture, entropy reduction, naked singularity

\section{INTRODUCTION}

The chronology protection conjecture is the first hypothesis proposed by Stephen Hawking that the laws of physics can prevent the propagation of time on a microscopic scale. The admissibility of time travel is mathematically represented by the existence of closed time-like curves in certain solutions of the general relativistic field equations. The Chronology protection conjecture should be different from the time check. Under the time check, each closed time-like curve passes the event range, which may prevent the observer from discovering causal conflicts (also known as time violations).

As we all know, in general relativity, in principle, space-time is a possible space-time travel, that is, some trajectories will form a cycle with time, and the observers who follow them can return to their past. These cycles are called closed space-time curves (CTC). There is a close relationship between time travel and the speed at which the speed of light (super speed of light) in vacuum is greater. In principle, in different Lorentz frames, the path is executed between two points at the speed of light, and then the return path is executed at the speed of light. In principle, it can even return to the origin before leaving.

In 1972, Press and Teukolsky[13] proposed that It is possible to add a mirror to the outside of a black hole to make a black hole bomb (according to the current explanation, this is a scattering process involving classical mechanics and quantum mechanics $[3,7,11,12])$.

When a bosonic wave is impinging upon a rotating black hole, the wave reflected by the event horizon will be amplified if the wave frequency $\omega$ lies in the following superradiant regime[13, 15, 16]

$$
0<\omega<m \Omega_{H}, \Omega_{H}=\frac{a}{r_{+}^{2}+a^{2}},
$$

where $m$ is azimuthal number of the bosonic wave mode, $\Omega_{H}$ is the angular velocity of black hole horizon. This

*Electronic address: wxchen4277@qq.com 
amplification is superradiant scattering. Therefore, through the superradiation process, the rotational energy of the black hole can be extracted. If there is a mirror between the black hole's horizon and infinite space, the amplified wave will scatter back and forth and grow exponentially, which will cause the black hole's superradiation to become unstable.

The cosmic censorship hypothesis has not been directly verified, and some physicists also question the validity of the cosmological censorship hypothesis. Through theoretical prediction, it pointed out that the existence of naked singularities is possible. The collapse of some stars with different structures and shapes is not necessarily accompanied by the event horizon as analyzed in the past. Examples include the example of the density-uneven stars proposed by the German physicist Hans Jürgen Seifert and his colleagues in 1973.

In general theory of relativity, naked singularity is a theoretically speculated singularity of gravity, which is not surrounded by the event horizon. A black hole is composed of the singular point of gravity and the event horizon that surrounds it. The fastest light cannot escape beyond the event horizon. Therefore, in theory, outside observers cannot directly observe the phenomenon inside the black hole. Naked singular points are the opposite. Light and other particles have the opportunity to escape from the singular point to a distance, and the event horizon does not exist; outside observers have the opportunity to observe the phenomenon of violently distorting time and space near the singular point. The existence of naked singularity is of great importance for astrophysics and other fields. One of them is the possibility of observing the collapse of stars into infinitely dense points. On the other hand, its existence and characteristics are a good opportunity to test the theory of quantum gravity.

From [6], $\mu=y \omega$, we get inequality (2)

$$
\frac{m a}{\frac{2 r_{-}^{2}}{y}+\left(M r_{-}-r_{-}^{2}\right) y+\left(y+\frac{1}{y}\right) \frac{a^{2}}{2 M}\left(M-r_{-}\right)}<\mu<\sqrt{2} m \Omega_{H},
$$

where $\mu$ is the mass. If there is no strong cosmic censorship conjecture, then there may be naked singularities. From [4][5][6],I found that the chronology protection conjecture may not hold.

\section{DESCRIPTION OF THE KERR-BLACK-HOLE SYSTEM}

The metric of the Kerr black hole[9, 10] (in natural unit $\mathrm{G}=\mathrm{c}=1$ ) is

$$
\begin{gathered}
d s^{2}=-\frac{\Delta}{\rho^{2}}\left(d t-a \sin ^{2} \theta d \phi\right)^{2}+\frac{\rho^{2}}{\Delta} d r^{2}+\rho^{2} d \theta^{2}+\frac{\sin ^{2} \theta}{\rho^{2}}\left[a d t-\left(r^{2}+a^{2}\right) d \phi\right]^{2} . \\
\Delta=r^{2}-2 M r+a^{2}, \rho^{2}=r^{2}+a^{2} \cos ^{2} \theta .
\end{gathered}
$$

We know the Klein-Gordon equation

$$
\left(\nabla^{\nu} \nabla_{\nu}-\mu^{2}\right) \Psi=0
$$

Eigenvalues of the above formula and spherical harmonic functions can be written as

$$
\Psi_{\operatorname{lm}}(\mathrm{t}, \mathrm{r}, \theta, \phi)=\sum_{\mathrm{l}, \mathrm{m}} \mathrm{e}^{\mathrm{im} \phi} \mathrm{S}_{\operatorname{lm}}(\theta) \mathrm{R}_{\operatorname{lm}}(\mathrm{r}) \mathrm{e}^{-\mathrm{i} \omega \mathrm{t}}
$$


Substituting (6) into the Klein-Gordon wave equation, we get that the angular function $\mathrm{S}_{\operatorname{lm}}(\theta)$ satisfies the following equation $[1,8,14]$

$$
\frac{1}{\sin \theta} \frac{d\left(\sin \theta \frac{d S_{l m}}{d \theta}\right)}{d \theta}+\left[K_{l m}+a^{2}\left(\mu^{2}-\omega^{2}\right) \sin ^{2} \theta-\frac{m^{2}}{\sin ^{2} \theta}\right] S_{l m}=0 ;
$$

For the references[1, 8, 14],we know inequality $(8)$

$$
K_{l m} \geq m^{2}-a^{2}\left(\mu^{2}-\omega^{2}\right),
$$

where $l$ is the spherical harmonic index, $m$ is the azimuthal harmonic index with $-l \leq m \leq l$ and $\omega$ is the energy of the mode.

\section{KERR-BLACK-HOLE-MASSIVE-SCALAR-FIELD SYSTEM AND SUPERRADIANT QUANTUM EFFECT}

The radial Klein-Gordon equation[8, 14] obeyed by $R_{l m}$ is given by

$$
\Delta \frac{d\left(\Delta \frac{d R}{d r}\right)}{d r}+U R=0
$$

where

$$
\Delta=r^{2}-2 M r+a^{2}
$$

and

$$
U=\left[\omega\left(\mathrm{r}^{2}+a^{2}\right)-\mathrm{m} a\right]^{2}+\Delta\left[2 \mathrm{~m} a \omega-\mu^{2}\left(\mathrm{r}^{2}+a^{2}\right)-K_{l m}\right] .
$$

The inner and outer horizons of the black hole are

$$
r_{ \pm}=M \pm \sqrt{M^{2}-a^{2}}
$$

and we know that

$$
r_{+}+r_{-}=2 M, r_{+} r_{-}=a^{2} .
$$

We use tortoise coordinate $r_{*}$ by equation $\frac{d r_{*}^{2}}{d r^{2}}=\frac{r^{2}}{\Delta}$ and another radial function $\psi=$ rR.We get the following equation

$$
\frac{\mathrm{d}^{2} \psi}{\mathrm{dr}_{*}^{2}}+V \psi=0
$$

where

$$
V=\frac{U}{r^{4}}-\frac{2 \Delta}{r^{6}}\left(M r-a^{2}\right) .
$$

Then we get the asymptotic solutions for the radial wave equation below

$$
r \rightarrow \infty\left(\mathrm{r}_{*} \rightarrow \infty\right) \Rightarrow R_{\operatorname{lm}} \sim \frac{1}{r} e^{-\sqrt{\mu^{2}-\omega^{2}} r_{*}},
$$




$$
r \rightarrow r_{+}\left(r_{*} \rightarrow-\infty\right) \Rightarrow R_{\operatorname{lm}} \sim e^{-i\left(\omega-m \Omega_{H}\right) r_{*}}
$$

When

$$
\omega^{2}-\mu^{2}<0
$$

there is a bound state of the scalar field.

When $\varphi=\Delta^{\frac{1}{2}} R$, radial potential equation(9) can be transformed into the following equation

$$
\frac{d^{2} \varphi}{d r^{2}}+\left(\omega^{2}-V_{1}\right) \varphi=0, V_{1}=\omega^{2}-\frac{U+M^{2}-a^{2}}{\Delta^{2}}
$$

It was previously proved[4]that, the no-hair theorem is not necessarily true under the superradiation quantum effect. The previous literature[2] indicates that the superradiation effect is a process of entropy subtraction. It has been previously [4][5][6] shown that the no-hair theorem is not necessarily true under the superradiation quantum effect. Previous literature[2] has shown that the superradiation effect is a process of entropy reduction. The superradiant instability of the black hole is actually a phenomenon that occurs when the entropy reaches the maximum for the boson enters the black hole.If there is no strong cosmic censorship conjecture, then there may be naked singularities. The decrease of the black hole entropy indicates that there is periodicity inside the black hole, and if the situation of the naked singularity is connected, then the chronology protection conjecture may not hold.

\section{THAT RESULT CONTAINED SOME CONCLUSIONS THAT VIOLATED THE CHRONOLOGY PROTECTION CONJECTURE}

For $\mu^{2}=y^{2} \omega^{2}$, when $y>4.352$, there exists a certain interval to let the inequality

$$
\frac{m a}{\frac{2 r_{-}^{2}}{y}+\left(M r_{-}-r_{-}^{2}\right) y+\left(y+\frac{1}{y}\right) \frac{a^{2}}{2 M}\left(M-r_{-}\right)}<\mu<\sqrt{2} m \Omega_{H},
$$

be satisfied. So the Kerr black hole is superradiantly stable at that time [6].

We see that when $y$ is very large, the superradiant stability interval of $\mu$ can always include some numerical solutions of the superradiant instability(The case under the no-hair theorem) obtained by previous people. For[4],at that time, the black hole entropy began to decrease.If there is periodicity inside the black hole and the naked singularity exists, then the chronology protection conjecture may not hold.

\section{SUMMARY}

This article puts forward a point that, under the quantum gravity equation, although there is no concept of wormholes, the chronology protection conjecture may not be true.

[1] Berti, E., Cardoso, V. and Casals, M. [2006], 'Eigenvalues and eigenfunctions of spin-weighted spheroidal harmonics in four and higher dimensions', Physical Review D 73(2), 024013.

[2] Brito, R., Cardoso, V. and Pani, P. [2015], 'Superradiance', Lect. Notes Phys 906(1), 1501-06570. 
[3] Cardoso, V., Dias, O. J., Lemos, J. P. and Yoshida, S. [2004], 'Black-hole bomb and superradiant instabilities', Physical Review D 70(4), 044039.

[4] Chen, W.-X. [2020a], 'The possibility of the no-hair theorem being violated', Available at SSRN 3569639 .

URL: https://dx.doi.org/10.2139/ssrn.3569639

[5] Chen, W.-X. [2020b], 'The strong cosmic censorship conjecture may be violated', SSRN Electronic Journal .

URL: https://dx.doi.org/10.2139/ssrn.3610419

[6] Chen, W.-X. and Huang, Z.-Y. [2019], 'Superradiant stability of the kerr black hole', International Journal of Modern Physics D.

URL: https://doi.org/10.1142/S0218271820500091

[7] Greiner, W., Müller, B. and Rafelski, J. [1985], Evolution of the vacuum state in supercritical potentials, in 'Quantum Electrodynamics of Strong Fields', Springer, pp. 257-299.

[8] Hartman, T., Song, W. and Strominger, A. [2010], 'Holographic derivation of kerr-newman scattering amplitudes for general charge and spin', Journal of High Energy Physics 2010(3), 118.

[9] Herdeiro, C. A. and Radu, E. [2014], 'Kerr black holes with scalar hair', Physical review letters 112(22), 221101.

[10] Kerr, R. P. [1963], 'Gravitational field of a spinning mass as an example of algebraically special metrics', Physical review letters 11(5), 237.

[11] Manogue, C. A. [1988], 'The klein paradox and superradiance', Annals of Physics 181(2), 261-283.

[12] Penrose, R. [2002], 'Gravitational collapse: The role of general relativity (reprinted from rivista del nuovo cimento, numero speziale, i, vol 257, 1969)', General Relativity and Gravitation 34(7), 1141-1165.

[13] Press, W. H. and Teukolsky, S. A. [1973], 'Perturbations of a rotating black hole. ii. dynamical stability of the kerr metric', The Astrophysical Journal 185, 649-674.

[14] Teukolsky, S. A. [1973], 'Perturbations of a rotating black hole. 1. fundamental equations for gravitational electromagnetic and neutrino field perturbations', Astrophys. J. 185, 635-647.

[15] Vilenkin, A. [1978], 'Exponential amplification of waves in the gravitational field of ultrarelativistic rotating body', Physics Letters B 78(2-3), 301-303.

[16] Zel'dovich, Y. B. [1972], 'Pis' ma zh. eksp. teor. fiz. 14, 270 (1971)[jetp lett. 14, 180 (1971)]', Zh. Eksp. Teor. Fiz 62, 2076. 